\title{
Polarization-domain-wall in a dual-color mode-locked fiber laser
}

\author{
A. Nady ${ }^{1,2}$, M. Kemel ${ }^{1}$, G. Semaan ${ }^{1}$, M. Salhi ${ }^{1}$, F. Sanchez $^{1}$ \\ 1. Laboratoire de Photonique d'Angers, Université d'Angers, E. A. 4464, 2 Boulevard Lavoisier, 49045 Angers, France \\ 2. Department of Physics, Faculty of Science, Beni-Suef University, 62511 Beni-Suef, Egypt
}

Generation of rectangular pulses in fiber lasers is of a great interest due to their applications in different fields. Dissipative soliton resonance and polarization domain-wall soliton [1,2] have been introduced as two different mechanisms for rectangular pulse formation.

In this work, we demonstrated domain-wall pulses in a dual-color Er-doped fiber laser. The dual-wavelength operation is realized through the intracavity birefringence induced spectral filtering effect. Stable domain-wall pulses are obtained via the cross-coupling between two lasing wavelengths. This is induced by $20 \mathrm{~m}$ high nonlinear fiber (HNLF) incorporated inside a ring cavity consisting of $6 \mathrm{~m}$ double-clad fiber Er:Yb and $13 \mathrm{~m}$ SMF. In order to study the pulse dynamics, we provide polarization-resolved measurements as well as wavelength-resolved measurements shown in Fig. 1. The optical spectrum shows two spectral lobes centered at $\sim 1547 \mathrm{~nm}$ and $\sim 1569 \mathrm{~nm}$, with wavelength separation of $\sim 22 \mathrm{~nm}$ while the the corresponding temporal trace exhibits a square pulse with an intensity dip, with repetition frequency of $\sim 5.1 \mathrm{MHz}$ corresponding to the cavity round-trip time. The wavelength-resolved measurements (Fig. 1(a)) show that the filtered emissions are two antiphase pulses and the laser emission was found to be interchanging between the two wavelengths; $1547 \mathrm{~nm}$ (black trace), and $1569 \mathrm{~nm}$ (red trace). This indicates that the total output signal is a combination of two wavelength domain-wall pulses and co-propagate as a non-dispersive unit with a single fundamental repetition rate. On the other hand, the polarization-resolved temporal traces (Fig. 1(b)) of the laser emission display two pulses in the two orthogonal polarization states represented by blue and red traces emerging from the total output signal which is represented by black trace and shows an intensity dip arising from the two orthogonal pulses overlap. Each of the two orthogonal pulses exhibits different pulse width with the same repetition rate. They trap each other through Kerr nonlinearity (cross-phase modulation) and travel as one unit constituting a domain wall.

Polarization-resolved optical spectra in which the signal comes from a single polarization direction are shown in Fig. 1(c) and indicate that each of the polarization components exhibits different spectral distributions and alternately dominates respectively the shorter and longer wavelength after polarization beam splitter. This different wavelength distribution indicates the incoherent coupling between the two polarization components [3]. The presentd results are different from our previous work [4], in which the formation mechanism of the intensity dip is due to the combined effect of gain depletion and chromatic dispersion induced difference in group velocity.

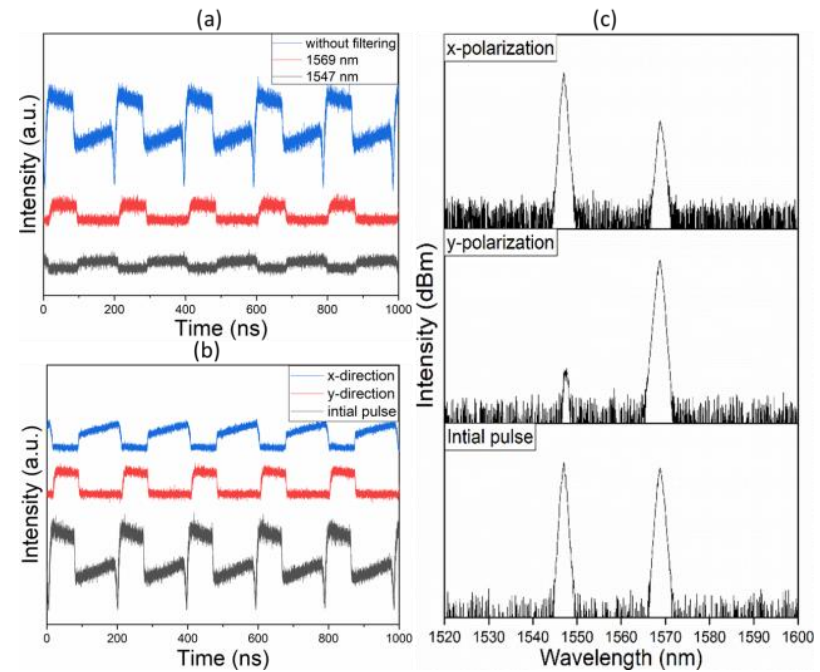

Fig. 1. The Oscilloscope trace of the initial and filtered emission (a), polarization resolved oscilloscope traces (b), and polarization resolved optical spectra (c).

\section{References}

[1] W. Chang, A. Ankiewicz, J. M. Soto-Crespo, and N. Akhmediev, "Dissipative soliton resonances," Phys. Rev. A. 78, 023830 (2008).

[2] P. Wang, K. Zhao, X. Xiao, and C. Yang, "Pulse dynamics of dual-wavelength dissipative soliton resonances and domain wall solitons in a Tm fiber laser with fiber-based Lyot filter," Opt. Express 25, 30708-30719 (2017).

[3] H. Zhang, D. Y. Tang, L. M. Zhao, and R. J. Knize, "Vector dark domain wall solitons in a fiber ring laser," Opt. Express 18, 4428-4433 (2010).

[4] Y. Meng, G. Semaan, M. Kemel, M. Salhi, A. Komarov, and F. Sanchez, "Color domains in fiber lasers," Opt. Lett. 43, 5054-5057 (2018). 